\title{
STANDARDIZATION OF LOWER EXTREMITY QUANTITATIVE LYMPHEDEMA MEASUREMENTS AND ASSOCIATED PATIENT-REPORTED OUTCOMES IN GYNECOLOGIC CANCERS
}

\begin{abstract}
Practice changing standardization of lower extremity lymphedema quantitative measurements with integrated patient reported outcomes will likely refine and redefine the optimal risk-reduction strategies to diminish the devastating limb-related dysfunction and morbidity associated with treatment of gynecologic cancers. The National Cancer Institute brought together a diverse group of cancer treatment, therapy and patient reported outcomes experts to discuss the current state-ofthe-science in lymphedema evaluation with the potential goal of incorporating new strategies for optimal evaluation of lymphedema in future developing gynecologic clinical trials. The key discussions are outlined in this report.
\end{abstract}

\section{INTRODUCTION}

The National Cancer Institute (NCI), Division of Cancer Prevention, hosted a workshop titled "Standardization of Lower Extremity Quantitative Lymphedema Measurements and Associated Patient Reported Outcomes in Gynecologic Cancers" on June 21, 2019. The workshop attendees represented a diverse group of investigators with expertise in breast and gynecologic lymphedema (LE) development and management, quantitative measurements of lymphedema, limb-related dysfunction, lymphedema preventative strategies, and symptom/quality-of-life endpoints. The goal was to evaluate the current state-of-the-science for optimal lower extremity quantitative lymphedema measurement and potential risk-reduction strategies, as well as, lower extremity limb-related dysfunction and symptom/quality of life endpoints, which may be incorporated into 
developing new clinical trials evaluating treatment-related lower extremity lymphedema (LEL) and lymphedema endpoints in gynecologic cancer.

LEL is an abnormal accumulation of fluid, which in gynecologic cancers occurs below the navel and can involve the bilateral legs and feet, the lower abdomen, bilateral hip area and genitals. LEL is a multifaceted disease of waxing and waning nature requiring quantitative objective measurements and qualitative subjective measurements, in addition to potential known risk factors (i.e., body mass index [BMI], age, race, and presence of pre-clinical LE) and treatment factors (i.e., sentinel lymph node dissection versus lymphadenectomy [LD], +/- radiation therapy, and +/chemotherapy) for full assessment.

Lack of standardization regarding the definition and quantification of LE has been an ongoing barrier limiting the ability to move knowledge in this field forward [1]; however, advancing understanding is critical due to the potential for progression. Given the non-linear relationship between symptoms and severity of LE, even those with minimal early swelling may be symptomatic, leading to increased morbidity and a costly burden on both patients and the healthcare system.

There has been more research on post-operative LE related to breast cancer compared to gynecologic cancers. Therefore, the incidence of LEL has been under-recognized with comparatively limited data on the physical and emotional impact of LEL [2]. However, many gynecologic cancer survivors are living with discomfort, disfigurement, disruption of activities, and diminished quality of life because of LEL. For some, this is further compounded by recurrent 
infections and hospitalizations that lead to the degradation of a patient's condition and eventual disability.

The ability to differentiate swelling from multiple pre-operative cofounders (i.e., obesity, venous stasis or insufficiency) and normal post-surgical swelling from the signs or symptoms of developing LE is critical to the proper assessment of LEL. To avoid the overestimation of LE incidence and diminish the risk of post-operative swelling being attributed to LE, a designated time period after surgery (i.e., three months) should not be factored into the LE analysis. Workshop participants also agreed peak incidence of the condition may be six to nine months; however, clinical trials should collect data for at least an 18-24 months post-surgery for complete assessment. Relatively fewer new incidences of LEL will occur after the 24 month post-operative period.

\section{MEASUREMENT OF LOWER EXTREMITY LYMPHEDEMA}

Four techniques have most often been employed to measure LE: (1) circumferential measurements (2) perometry, (3) water displacement, and (4) bioimpedance spectroscopy (BIS), with perometry and BIS largely restricted to the upper extremities in widespread usage thus far. Water displacement, due to impracticality and potential for imprecision, has largely been replaced by circumferential measurements via tape measures. However, these measurements can be imprecise and present challenges when standard protocols are not followed.

In most clinical practices and the preponderance of studies, $a \geq 10 \%$ change from baseline is used as the standard indicator of the presence of LE. Yet controversy remains on the definition (limb 
volume change $\geq 5 \%$ vs. $10 \%$ ), and the optimal quantitative measurement. Circumferential measurements remain the standard comparative measurement, although variation in the way the method is performed and assessed can lead to a lack of reproducible data and ambiguity.

The challenge of sequential measurements came to light in Gynecologic Oncology Group (GOG) 244, the Lymphedema and Gynecologic Cancer Study (The LEG Study), a multi-institutional study of 1,054 women newly diagnosed with endometrial, cervical, and vulvar cancer who received surgery with a lymphadenectomy (LD) as primary intervention [3]. The study was designed to prospectively evaluate the incidence of, and potential risk factors for, LEL. Here, leg volume was used as a surrogate for LE and calculated from measurements obtained at $10-\mathrm{cm}$ intervals starting $10 \mathrm{~cm}$ above the bottom of the heel to the inguinal crease. A baseline assessment was taken within 14 days prior to surgery, followed by assessments at four to six weeks and 3, 6, $9,12,18$, and 24 months post-operatively. LE was defined as a limb volume change (LVC) $\geq 10 \%$ from baseline.

Limb volume was calculated as a summation of the multiple truncated cones measured in the leg using the formula for a truncated cone:

$$
\mathrm{V}=(\mathrm{h})\left(\mathrm{C}^{2}+\mathrm{Cc}+\mathrm{c}^{2}\right) / 12(\pi)
$$

( $\mathrm{h}=$ height of segment; $\mathrm{C}=$ circumference at top of segment; $\mathrm{c}=$ circumference at bottom of segment) 
LVC was recorded as the difference in summation of the truncated cones limb volume over time, with incidence of $\mathrm{LVC} \geq 10 \%$.

Of the 1,054 women enrolled, 914 were evaluable for LVC, including 734 endometrial, 138 cervical, and 42 vulvar patients. The incidence of $L V C \geq 10 \%$ for these cancers was $34 \%(\mathrm{~N}=247)$, $35 \%(\mathrm{~N}=48)$, and $43 \%(\mathrm{~N}=18)$, respectively. The peak incidence of LE was at the four-to-six-week assessment, which may be attributable to post-surgical swelling. Regression analysis showed a decreased risk with advanced age $(p=0.0467)$ and an increased risk with a node count $>8(p=0.033)$ $[3]$.

The same protocol was carried out across 52 enrolling parent institutions and 72 affiliates, with some associates shared across sites. Despite a rigorous training program required for all sites prior to enrolling patients, user error appears to have contributed to a significant discrepancy in the data. Of the evaluable patients, $32 \%$ had some variance in leg length, which required truncation to the longest length with consistent measurements across all intervals for follow-up. The protocol also allowed up to a $1.0 \mathrm{~cm}$ variation in each of the paired measurements; $11.3 \%(\mathrm{~N}=119)$ exceeded the threshold. Of those 119 patients, 10 exceeded $1.5 \mathrm{~cm}$ and were excluded from final analysis.

Serial circumferential measurements were found to be tedious and labor intensive. Researchers also experienced a high loss to follow-up rate over the study's two-year period ( $48 \%$ cervical; $54 \%$ endometrial; $40 \%$ vulvar), partially attributable to inconsistent patient counseling across institutions. Concerns regarding some of the data elements may now challenge the study's 
conclusions. The LEG Study's highlighted these challenges that ultimately lead to the NCI workshop.

Significant attrition in measurements and survey completion over the course of the study also impacted results in a smaller-scale study focused on endometrial cancer. That study piloted the use of an objective measurement to prospectively evaluate the incidence of LEL after minimally invasive surgery for endometrial cancer staging, specifically in LD versus sentinel lymph node biopsy (SLNB) [4]. In comparison with the LEG Study, which employed many associates across multiple sites, this study used only one coordinator trained by a certified LE specialist.

Each of these studies demonstrates the importance of protocol training, adherence to protocol requirements and the importance of obtaining preoperative baseline measurements, which are essential to determine changes in the condition over time.

\section{OBJECTIVE MEASUREMENT: BIOIMPEDANCE SPECTROSCOPY (BIS) AND EMERGING TECHNOLOGIES}

Over the past three decades, BIS has increasingly been used to screen for breast-cancer-related LE [5]. The National Lymphedema Network [6] position paper on screening and measurement for early detection of breast-cancer-related LE supports the use of BIS as an alternative to circumferential tape measurements. Yet, the Breast Cancer Evaluation Database to Guide Effectiveness Task Force rated BIS, circumference measurement, and water displacement each as Level 4 (highly recommended) due to good reliability, validity, and clinical utility [7]. 
BIS technique shows potential for adoption as a complementary measure to the lower extremities; however, few clinical trials exist on this use for BIS, and further research is needed to determine its utility in LEL related to gynecologic cancers. The underlying principle of BIS for LE detection is simple: The opposition (impedance or resistance) to the flow of a harmless alternating electric current through the body is quantitatively related to the extracellular fluid (ECF) volume. Since lymph is a principal component of ECF, a change in resistance of a limb at risk of LE is indicative of a change in lymph volume. The relationship between resistance and volume is inverse such that, an increase in lymph occurs in early-stage LE and is indicated by a decrease in measured resistance.

While it is possible to convert resistance, which is measured in ohm, to an absolute volume in $\mathrm{mL}$, this requires invoking assumptions that are not readily proven to be correct in LE. Alternatively, in unilateral LE of a limb, the resistance of the limb at risk of or affected with LE is compared to the resistance of the contra-lateral unaffected limb-a method analogous to that used for circumferential measurement. This comparison is expressed as a ratio of the resistance of the unaffected limb to the resistance of the affected limb. Presence of LE is indicated when this ratio exceeds a threshold determined from the normal distribution of ratios seen in a healthy population. Typically, detection thresholds are set at mean +2 standard deviations (SD) or mean $+3 \mathrm{SD}$. A simplified metric is also used, L-Dex, which linearizes ratios such that an L-Dex score of 10 units is equivalent to the mean $+3 \mathrm{SD}$ threshold [8]. Clinically, an absolute L-Dex greater than 10 or a change in L-Dex score $>10$ is indicative of the presence of LE. Considering the building body of evidence, it may be more appropriate to use $<+3 \mathrm{SD}$ threshold (i.e., $+2 \mathrm{SD}$, which is equivalent to L-Dex of 6.5 or 7) to increase the sensitivity and specificity of detection, however this requires 
the accumulation of maturing data to make this conclusion [9]. Due to fluctuations inherent to the condition, a "positive" BIS result should be considered only if two consecutive qualifying values are recorded approximately one week apart.

In a number of sensitivity and specificity studies comparing BIS against other detection modalities for breast-cancer-related LE, such as volume-either from perometry or circumferences-or presence of LE confirmed by imaging techniques (including lymphoscintigraphy and indocyanine green), specificity has been greater than $95 \%$ with sensitivities of $80 \%$ to $100 \%$. However, significant levels of false positives have been reported with BIS, and many patients with abnormal L-Dex scores do not go on to develop clinical LE. In Barrio et al., [10], of 25 patients with abnormal L-Dex scores ( $>10$ or +10 increase from baseline), only four patients progressed to LE. Most patients who developed LE did not have an abnormal L-Dex result in advance of diagnosis. Sensitivity in this study was $75 \%$. In a large, multisite study in the UK $(\mathrm{N}=1,000)$ comparing BIS and perometry, many false positives were found with BIS [11]. Other studies have found lower sensitivity, including $<50$ in Lahtinen et.al., [12].

The current data suggests that a LE diagnosis should not be based on BIS measurement alone and should include at least one other complementary validated method. Studies determining the effectiveness of BIS for early intervention must include a control group that does not receive the intervention, and sufficient follow-up (12-24 months, minimum) to observe the natural progression of L-Dex scores in absence of and in response to treatment. 
BIS is most beneficial for identifying LE at the latent (stage 0) phase and has been shown, in some patients, to detect the presence of the condition a mean of 10 months before a clinically defined presence had been established [8]; however, other studies suggest more data is still needed $[10,13]$.

More recently, MRI has been studied as another noninvasive technique for imaging lymphatics $[14,15,16,17]$. These developing Magnetic Resonance Imaging (MRI) techniques and other emerging technologies such as Tissue Dielectric Constant (TEC) appear promising for evaluating LEL $[18,19]$; however, further evaluation of associated cost and risk is necessary before these technologies can be implemented in large, multicenter trials.

\section{SLNB - VALUE IN GYNECOLOGIC CANCER}

At the core of advancing prevention is the need to evaluate the benefits and risks of SLNB in both cervical and endometrial cancer. Based on our current knowledge, we must investigate whether SLNB can become the standard of care by improving quality of life without impacting recurrence or survival.

Typically, patients with clinical stage I endometrial cancer have a good prognosis and are at low

risk for lymph node metastasis. The primary role of lymphadenectomy (LD) in the treatment of endometrial cancer is to identify the small subset of patients with lymph node metastasis at higher risk for disease-related mortality. Although surgical staging has been the norm for decades, two prospective randomized trials evaluating the benefit of $\mathrm{LD}$ at the time of hysterectomy failed to demonstrate a survival advantage while confirming significant LE-related morbidity with the procedure $[20,21]$. 
For women with early-stage cervical cancer (stages IA and 1-IB1), the pathologic status of the lymph nodes is the most important prognostic factor and guides post-operative adjuvant therapy. However, the incidence of nodal metastases in women with early-stage cervical cancer is only $10 \%-15 \%$, meaning that $85 \%-90 \%$ of patients undergo a complete pelvic LD - a procedure associated with increased blood loss, neurovascular and ureteral injuries, infections, LE, lymphocyst formation, and venous thromboembolism - with no clinical benefit. To decrease the number of patients undergoing complete $\mathrm{LD}$, and thereby decrease complications from complete LD at all sites, lymphatic mapping and SLNB have been established as a standard in multiple solid tumors.

Numerous studies have shown lymphatic mapping and SLNB for women with early-stage cervical cancer to be both safe and valid. In the SENTICOL study—a large, prospective, multi-institutional investigation where SLNB was performed, followed by complete pelvic LD in the treatment of women with early-stage cervical cancer-23 of 25 patients with metastatic disease to the pelvic lymph nodes had positive sentinel lymph nodes (SLN) [22]. In one of the two patients with a "false negative," a metastatic node was detected in a pelvis that did not map. Today, most authorities would not consider that a true false negative, as a non-mapping hemi-pelvis is an indication for a complete ipsilateral LD.

More recently, a large retrospective study determined that sensitivity is high for detecting lymph node metastases with a low negative predictive value. Results showed a sensitivity of $96.4 \%$ and a negative predictive value of $99.3 \%$ in 188 patients with early-stage cervical cancer who 
underwent SLNB followed by complete pelvic LD [23]. A recent meta-analysis showed a pooled sensitivity of $94 \%$ for the technique. Furthermore, when limiting the patient population to women with tumors $<4 \mathrm{~cm}$ in size with no evidence of metastatic disease on preoperative imaging or intraoperative assessment and bilateral sentinel nodes detected, the negative predictive value reaches $99.92 \%$ [24]. That is, if the SLNs were negative, the risk of there being undetected nodal disease was only $0.08 \%$.

When evaluating the technique for oncologic outcomes, there was no difference in recurrence-free survival (RFS) between the approaches (two and five-year survivals of 97\% and 93\% for 110 women with cervical cancer who underwent SLNB only compared to $95 \%$ and $92 \%$ for the 1,078 similar women who had complete LD, respectively). Furthermore, multivariate analysis demonstrated no difference in the hazard ratio for recurrence between the two approaches.

Other studies on SLNB in cervical cancer have similarly suggested that the procedure is highly sensitive and specific, associated with less morbidity and LE, and cost-effective. Completed in 2012, SENTICOL II was a randomized study of SLNB + pelvic LD versus SLNB only, evaluating 206 patients for morbidity and quality of life. No cases of false negative SLNs were identified, and the surgical morbidity and major morbidity related to LD were both reduced with SLNB only ( $31 \%$ vs. $52 \%$ and $1 \%$ vs. $6 \%$, respectively). Analysis of the quality of life (SF36) questionnaires demonstrated significantly lower scores for the LD patients, while analysis of leg LE showed lower circumferences in the SLNB group. Leg heaviness and leg fatigue were significantly worse in the LD group [25]. 
Despite evidence that SLNB is valid and provides equivalent oncological outcomes for women with cervical cancer, there are still few studies showing a decrease in post-operative morbiditythe most commonly stated rationale. In other cancer sites, changing standard of care from complete regional LD to SLNB has decreased LE and other operative morbidity. For example, LE in women with breast cancer decreased after surgeons moved from complete axillary LD to SLNB. Niikura et al., [26] reported on 35 patients with stages IA-IIA cervical cancer that underwent radical hysterectomy and SLNB only (if negative). Twenty-three underwent SLNB alone without systematic pelvic LD, and none diagnosed with negative SLNs experienced a lymph node recurrence in the pelvis. New symptomatic lower extremity LE was identified in two (9\%) of the 23 patients who underwent SLNB alone and five (42\%) of 12 patients who underwent systematic LD.

Are we more accurate at identifying lymph node metastases with SLNB than a typical pelvic lymph node dissection (PLND) in cervical cancer? The data suggest we are. A retrospective study showed 17\% lymph node metastases with SLNB versus 7\% with PLND, where SLNB to PLND patients were matched 3:1 for tumor size $( \pm 5 \mathrm{~mm})$, histology, depth of invasion $( \pm 2 \mathrm{~mm})$, and presence of capillary lymphatic space invasion [27]. Survival curves were the same.

As for whether SLNB can replace PLND for early cervical cancer, consider a retrospective evaluation to evaluate RFS and short-term morbidity in patients with early cervical cancer who underwent bilateral pelvic lymphadenectomy (BPLND) versus bilateral sentinel lymph node biopsy only (BSLNB) at primary surgery. A total of 1,188 node-negative patients were identified (BPLND, 1078; BSLNB, 110). Results showed no difference between BPLND and BSLNB in 
two- and five-year RFS (95\% vs. $97 \%$ and $92 \%$ vs. $93 \%$, respectively); tumor size; histology; invasion depth; intra-operative complications; or short-term morbidity. BPLND was associated with increased surgical time (2.8 vs. $2.0 \mathrm{~h}, \mathrm{p}<0.001)$; greater blood loss $(500 \mathrm{~mL}$ vs. $100 \mathrm{~mL}, \mathrm{p}$ $<0.001)$; higher rates of transfusion ( $23 \%$ vs. $0 \%, \mathrm{p}<0.001)$; and more post-operative infection $(11 \%$ vs. $0 \%, \mathrm{p}=0.001)[28]$.

In patients with apparent early-stage endometrial cancer, SLN mapping has been proposed as an alternative lymph node assessment strategy to LD. The goal is to accurately identify lymph node metastasis while saving patients from the morbidity of complete LD. The utility of this technique as a strategy to reduce overtreatment has been firmly established in other disease sites (breast cancer and melanoma). Two large prospective studies in patients undergoing SLN mapping followed by pelvic LD have reported a high SLN detection rate ( $85 \%-95 \%)$ and low false negative rate $(\sim 2.5 \%)$ in more than 500 patients with endometrial cancer [29,30]. Endometrial cancer is vulnerable to LE because patients tend to be obese, paired with other factors that impact venous return and post-operative radiation use. Node size is important for predicting survival in endometrial cancer, and patients with nodal metastasis have inferior survival, which has long been the catalyst for LD. Here, as with cervical cancer, research shows there is no survival advantage with lymph node dissection, as demonstrated in a randomized trial of 514 patients with stage I endometrial cancer. Although results found a $13 \%$ vs. $3 \%$ rate of LN metastasis [20].

High-quality data for sensitivity and false negative rate for SLN mapping comes out of the FIRES trial, where 385 patients with endometrial cancer underwent SLN mapping with indocyanine green 
(ICG), followed by LD, either pelvic (340) and/or para-aortic (196). The results showed a false negative rate related to SLN of 3\% and little related surgical morbidity $(0.3 \%)$ [30].

Evidence shows that the risk of LD outweighs the benefit; however, a survey from 2016 revealed only $4.5 \%$ of patients had SLN mapping for endometrial cancer in the U.S. Slow adoption of SLN mapping as an alternative suggests a lack of quality trials for the procedure and concern about the accuracy and potential benefit of the technique. The absence of a survival advantage with complete LD and the low false negative rate of SLN mapping support the assumption that a randomized trial comparing SLN mapping with LD is unlikely to demonstrate a clinically meaningful survival difference between these approaches, even with very large sample sizes. However, the value of SLN mapping as a tool to reduce the risk of LE and its consequences remains an important facet of the procedure requiring validation.

\section{PATIENT-REPORTED OUTCOME ASSESSMENT TOOLS}

In addition to quantitative measurements, physical examination, symptoms, and patient-reported outcomes (PRO) all contribute to the initial diagnosis and progression of LE.

For a PRO instrument to be effective, it must be brief, simple to complete, easy to score, and cover content consistent with clinical understanding of the signs and symptoms commonly reported in patients with LEL.

The Lymphedema Symptom Intensity and Distress Survey-Lower Limb (LSIDS-L) is a brief assessment tool that evolved from an upper limb tool by the Vanderbilt University School of 
Nursing [31]. Easily administered with REDCap ${ }^{\circledR}$ (Vanderbilt University) and other electronic capture systems, it can be completed in a healthcare provider's waiting room to monitor symptoms that occur over time. It was not designed to screen for LE.

To establish the measurement characteristics of the LSIDS-L and identify and compare symptoms associated with LEL across groups, Vanderbilt created a two-phase development study looking at patients with primary and secondary LE related to cancer and other trauma, as well as unknown LE. In study one, 277 individuals with LEL completed a 36-item draft LSIDS-L and demographics form. In study two, 64 more individuals with LEL, as well as 111 healthy controls, completed the items, as well as the Functional Assessment Screening Questionnaire, Profile of Mood States-Short Form, and Marlowe-Crowne Social Desirability Scale Short Form C. Analyses included descriptive statistics, statistical clustering of the LSIDS-L items, group comparisons, and correlations among measure scores. Clustering of the LSIDS-L items resulted in the removal of five items. The remaining 31 items clustered into eight areas: activity, soft tissue sensation, pain, resources, biobehavioral, neurological sensation, function, and sexuality.

Results showed six broad issues in greater than $66 \%$ of all groups, regardless of origin of swelling:

- Appearance concerns

- Decreased physical activity

- Fatigue

- Swelling

- Heaviness 
- Tightness

Highest intensity issues by burden score:

- Insurance frustration

- Lack of confidence in insurance

- Concerns about appearance

- Lack of interest in sex

- Partner's lack of interest in sex

- Gave up hobbies or leisure activities

Issues reported as causing patients the most distress:

- Insurance frustration

- Lack of confidence in insurance

- Partner's lack of interest in sex

Analysis indicated that severity and distress scores in this lower limb study were far more severe than in the team's previous upper limb studies. Researchers determined that the LSIDS-L is a valid self-report instrument for patients with LEL and intend to use the large amount of data collected to look at a general LE symptom profile that is not site specific to generate a subscale for individual regions based on symptoms that did not cross over. 
The Gynecological Cancer Lymphedema Questionnaire (GCLQ) is a modification of the validated Lymphedema Breast Cancer Questionnaire, which has shown to have predictive value in identifying patients at risk for early onset upper extremity LE $[32,33,34]$. Designed to be used in a clinical setting for screening and/or prevention or intervention, the GCLQ has 20 questions assessing 20 symptoms associated with LEL, which can be grouped into seven symptom clusters: aching, heaviness, infection-related, numbness, physical functioning, swelling (general), and swelling (limb). The GCLQ also includes supplemental items documenting patients' awareness of LEL diagnosis and any utilization of LE-specific treatment.

A validation study was conducted to determine the feasibility and efficacy of using the GCLQ as a symptom assessment to identify LEL. Twenty-eight gynecologic cancer survivors with documented LEL and 30 without a history of LE completed the GCLQ and provided feedback about their satisfaction with and the feasibility of using it at their oncology follow-ups. Scores differed significantly by LE diagnosis, as LEL patients had higher scores ( $\mathrm{p} 0.01)$. The large area under the curve (AUC) of 0.95 (95\% CI: 0.90-1.000) supports the conclusion that the GCLQ can distinguish between patients with and without LEL. The GCLQ was also easily understood by most participants $(95 \%, \mathrm{~N}=55 / 58)$. Overall, patients showed a high willingness $(96 \%, \mathrm{~N}=56 / 58)$ to complete the questionnaire, and many LEL patients $(88 \%, \mathrm{~N}=25)$ found it to be helpful in identifying symptoms. A clinical cutoff score of $\geq 4$ had distinguished between gynecologic cancer survivors with and without LE by demonstrating good sensitivity, specificity, and high overall accuracy percent, AUCs, and kappas (measure of diagnostic agreement) [35]. 
In GOG 244, the GCLQ was administered to all patients at the time of LVC measurement to explore whether patient self-reported symptoms are associated with a diagnosis of LEL and LVC, in addition to examining the survey's predictive value. Changes in the GCLQ symptom clusters and total score for those with and without a LVC $\geq 10 \%$ at the time leg volume was measured. After adjusting for assessment time and disease sites, the change in symptoms of swelling in general $(p<0.001)$, heaviness $(p=0.005)$, infection-related symptoms $(p=0.002)$, physical functioning $(\mathrm{p}=0.006)$, and GCLQ total score $(\mathrm{p}<0.001)$ were associated with an LVC $\geq 10 \%$. By disease site, in the endometrial group, symptoms of swelling in general $(\mathrm{p}<0.001)$, heaviness $(p<0.001)$, infection-related symptoms $(p=0.013)$, numbness $(p=0.01)$, and physical functioning $(p=0.002)$ were associated with an $L V C \geq 10 \%[36]$.

The association between the GCLQ total score and patient-reported LEL was also examined. The percentage of patients whose GCLQ total score increased $\geq 4$ from baseline was significantly associated with a patient-reported lymphedema diagnosis for the total sample $(\mathrm{p}<0.001)$, and each of the three cancers independently. An incremental change in the GCLQ total score was significantly associated with an LEL diagnosis in patients with cervical cancer $(\mathrm{p}<0.001)$, endometrial cancer $(p<0.001)$, and vulvar cancer $(p=0.015)[36]$.

The GCLQ symptom clusters were also analyzed. After adjustment for disease sites and assessment time points, the changes from baseline in all symptom cluster scores and GCLQ total score differed significantly and were able to distinguish between for those with and without patient-reported LE. In endometrial cancer patients, symptoms of aching $(p=0.001)$, swelling (general) $(\mathrm{p}<0.001)$, heaviness $(\mathrm{p}<0.001)$, infection $(\mathrm{p}<0.001)$, numbness $(\mathrm{p}<0.001)$, and physical 
functioning $(p<0.001)$ were significantly different between those diagnosed with and without LLE; specifically, women with lower extremity lymphedema were more symptomatic. The symptom cluster of swelling in the leg $(\mathrm{p}=0.03)$ had a mild group difference. For the cervical cancer group, symptoms of swelling (general) $(\mathrm{p}<0.001)$, heaviness $(\mathrm{p}=0.006)$, swelling in the leg $(\mathrm{p}=0.009)$, and numbness ( $\mathrm{p}=0.013$ ) showed a strong significant difference, and a marginal difference for infection $(\mathrm{p}=0.046)$. In women with vulvar cancer, symptoms of swelling (general) $(\mathrm{p}<0.001)$, heaviness ( $p=0.006)$, swelling in the leg $(p=0.01)$, and numbness $(p<0.001)$ were significantly different between those with and without LEL [36].

The GCLQ performed consistent with the findings and predictive value seen with the LBCQ used to evaluate LE symptoms of the upper extremities. The study results demonstrated that an incremental change of $\geq 4$ in the GCLQ total score prior to the first LLE diagnosis was significantly associated with a patient-reported diagnosis of LLE at the next time point for the total sample $(\mathrm{p}<0.001)$. By disease site, an incremental change in the GCLQ total score prior to the patientreported diagnosis of LEL was significantly associated with LEL at the next time point for patients with cervical cancer $(\mathrm{p}=0.002)$ and endometrial cancer $(\mathrm{p}=0.002)$ [36].

The GCLQ proved to be a simple, reliable and feasible tool that could be easily incorporated into the clinical setting to monitor for potential LEL symptoms. An increase in the GCLQ total score (indicating more LEL symptoms) within the two-year window when patients are most likely to develop LE could trigger measurement and evaluation, allowing healthcare providers to triage for early intervention. A consideration that may limit the predictive value of the GCLQ and PROs in general, however, is the patients' varying levels of awareness about their condition. Workshop 
participants cited patients who appear to have stage II LE upon gross observation, but self-report as not experiencing swelling. Greater education and more nuanced terminology (i.e., asking if they are experiencing "heaviness" or "discomfort" versus "symptoms of lymphedema") may go a long way toward making patients more cognizant of changes and optimizing PRO results. Continued monitoring, including self-measuring, and reporting of changes is important because although patients with large volume may not necessarily experience or be bothered by symptoms, they are at high risk for cellulitis, recurrent infections, hospitalizations, and other negative effects. With serial assessment and education regarding normal post-operative swelling, patients may be more fully aware of what is important to report.

The LEL Screening Questionnaire was developed at Mayo Clinic with attention to the tool's ability to detect LEL in both normal weight and obese patients following surgery for endometrial cancer [37]. It has been translated into Korean, German, and French, with additional translations planned, and is currently being used in the Women's Health Initiative Life and Longevity After Cancer Study [38], SENTICOL III (French only), multiple observational studies at Mayo Clinic and Memorial Sloan Kettering Cancer Center, and one planned international trial.

The ability of a screening tool to discriminate swelling from LEL versus adipose is an important characteristic given the prevalence of obesity in this patient population. The self-report LEL screener was developed with extensive input from stakeholders, including five physicians and five physical therapists with expertise in the management of LE and five patients with known diagnoses of LEL. 
Existing upper-extremity surveys were reviewed for relevant content and four approaches to questions were initially explored:

1. Absolute extent of signs/symptoms ("the skin on my leg feels tight"). If the sign/symptom was experienced on both sides of the body, respondents were directed to report about the side affected most.

2. Relative extent of signs/symptoms - a common approach in upper extremity LE measures in which respondents compare one side of the LE to the other ("one of my shoes feels tighter than the other").

3. Severity of swelling in three graphically depicted sections of the lower extremity.

4. Ability to see anatomical landmarks (hip bones, kneecap, ankle bone, bones, and veins on tops of feet).

Following the stakeholder's review, anatomical landmark questions were dropped due to concern over an inability to distinguish LE from adipose. Reviewers also suggested revising the graphical depiction (Set 3) to delineate four sections and adding one question on swelling in the genital area.

The remaining question sets were administered to roughly equal numbers of normal weight, overweight, obese, and morbidly obese women -88 with LEL and 39 without. Questions not statistically significantly associated with LEL were dropped unless deemed critical for content coverage. The rating scale for individual questions ranges from 0 (not at all) to 4 (very much), with the total score ranging from 0 to 52 . 
Receiver operating characteristic curve analysis was used to estimate an optimal cut score of 5 or more points for detecting LEL based on the AUC. For all participants, the sensitivity and specificity were $95.5 \%$ and $86.5 \%$, respectively, and the positive and negative likelihood ratios (LR+/LR-) were 7.1 and 0.05, respectively. For participants with BMI $\geq 30 \mathrm{~kg} / \mathrm{m} 2$, Sensitivity $=$ $94.8 \%$, Specificity $=76.5 \%, \mathrm{LR}+=4.0, \mathrm{LR}-=0.07$

A consideration in incorporating any symptom measurement tool is whether the goal is indication of initial diagnosis or detection of the progression of the condition's severity over time. Ideally, an assessment tool should be adaptive and quickly identify that the patient is headed down the wrong path at a point when their LE is reversible, in addition to including indicators reflective of latter stages of the condition. It is not clear which of the PROs described here should be used in a given setting or for a given purpose without empirical data from a comparative effectiveness study.

\section{SUMMARY AND FUTURE GOALS}

Research to date and the practical clinical experience of experts in attendance at the workshop, points strongly toward the need for standardization of optimal LEL quantitative measurements and baseline measurements prior to surgical intervention, as well as, the integration of PROs into gynecologic oncology protocols. Findings across sites and studies show potential for (a) improvements in protocol adherence and patient compliance to protocol measurements, (b) preoperative baseline assessments of LEL, (c) BIS as an objective, complementary technique for early detection of LEL, (d) the value of combination of LEL measurement techniques, including new emerging LE measurement technologies, to improve the outcome measurement power, (e) the 
use of sentinel lymph node mapping, and (f) the incorporation of PRO tools. However, before a full recommendation is made, more data are required to comprehensively understand the potential and challenges associated with each. The next step for NCORP Research Bases and gynecologic oncologists is the development of new, multisite clinical trials evaluating the findings from this gynecologic oncology LEL workshop's presentations and discussions. With the observations gained and LEL clinical trial development in gynecologic cancer patient, the evidence necessary for a new standard of care with improved quality of life for patients undergoing gynecologic cancer treatment can be established in the near future. 
AUTHOR CONTRIBUTIONS:

Study concept and design: All authors

Provision of materials or patients: All authors

Acquisition of data: All authors

Analysis and interpretation of data: All authors

Manuscript writing: All authors

Critical review of the manuscript: All authors

Final approval of manuscript: All authors 


\section{REFERENCES}

1.) O’Toole J, Jammallo LS, Miller CL, Skolny MN, Specht MC, Taghian AG. Screening for breast cancer-related lymphedema: the need for standardization. The Oncologist, 2013; 18(4): 350352. https://doi: 10.1634/theoncologist.2012-0387

2.) Bankhead C. Lower leg lymphedema common after gyn surgery. MedPage Today. 2018.

\section{https:/www.medpagetoday.org/meetingcoverage/sgo/72069}

3.) Carlson JW, Kauderer J, Hutson A, Carter J, Armer J, Lockwood S, Nolte S, Stewar, BR, Wenzel L., Walker J, Fleury A, Bonebrake A, Soper J, Mathews C, Zivanovic O, Richards WE, Tan A, Albert DS, Barakat RR. GOG 244 - The lymphedema and gynecologic cancer (LEG) study: Incidence and risk factors in newly diagnosed patients. Gynecol Oncol. 2020; 256(2): 467-474.

\section{https://doi: 10.1016/j.ygyno.2019.10.009}

4.) Watson CH, Lopez-Acevedo M, Broadwater G., Kim AH, Ehrisman J, Davidson BA, Lee PS, Valea F, Berchuck A, Havrilesky LJ. A pilot study of lower extremity lymphedema, lower extremity function, and quality of life in women after minimally invasive endometrial cancer staging surgery. Gynecologic Oncology, 2019;153(2):399-404. https://doi:

\subsection{6/j.ygyno.2019.02.021}

5.) Ward L, Winall A, Isenring E, Hills A, Czerniec S, Dylke E, Kilbreath S. Assessment of bilateral limb lymphedema by bioelectrical impedance spectroscopy. International Journal of Gynecological Cancer, 2011; 21(2): 409-418. https://doi.org/10.1097/IGC.0b013e31820866e1

6.) National Lymphedema Network. Screening \& early detection of breast cancer-related lymphedema. 2011. https://ymphnet.org/position-papers 
7.) Perdomo M, Davies C, Levenhagen K, Ryans K. Breast Cancer Edge Task Force Outcomes: Assessment measures of secondary lymphedema in breast cancer survivors. Rehabilitation Oncology, 2014;32(1): 22-35.

8.) Cornish BH, Chapman M, Hirst C, Mirolo B, Bunce IH, Ward LC, Thomas BJ. Early diagnosis of lymphedema using multiple frequency bioimpedance. Lymphology, 2001; 34(1): 2-11.

9.) Fu MR, Cleland CM, Guth AA, Kayal M, Haber J, Cartwright F, Kleinman R, Kang Y, Scagliola J, Axelrod D. L-dex ratio in detecting breast cancer-related lymphedema: reliability, sensitivity, and specificity. Lymphology.2013; 46(2):85-96.

10.) Barrio AV, Eaton A, Frazier TG. A prospective validation study of bioimpedance with volume displacement in early-stage breast cancer patients at risk for lymphedema. Annals of Surgical Oncology, 2015;22(Suppl. 3): 370-375. https://doi.org/10.1245/s10434-015-4683-0

11.) Bundred NJ, Stockton C, Keeley V, Riches K, Ashcroft L, Evans A, Skene A, Purushotham, A, Bramley M, Hodgkiss,T. Investigators of BEA/PLACE studies. Comparison of multifrequency bioimpedance with perometry for the early detection and intervention of lymphoedema after axillary node clearance for breast cancer. Breast Cancer Research Treatment, 2015;151(1):121-9. https://doi: 10.1007/s10549-015-3357-8.

12.) Lahtinen T, Seppälä J, Viren T, Johansson K. Experimental and analytical comparisons of tissue dielectric constant (TDC) and bioimpendance spectroscopy (BIS) in assessemnt of early arm lymphedema in breast cancer patients after axillary surgery and radiotherapy. Lymphatic Resarch and Biology, 2015; 13(3): 176-185. https://doi.org/10.1089//rb.2015.0019

13.) Qin ES, Bowen M.J, Chen WF. Diagnostic accuracy of bioimpedance spectroscopy in patients with lymphedema: a retrospective cohort analysis. Journal of Plastic, Reconstructive and Aesthetic Surgery. 2018; 71(7):1041-1050. https://doi: 10.1016/j.bjps.2018.02.012 
14.) Donahue M J, Donahue PC, Rane S, Thompson CR, Strother MK, Scott AO, Smith, SA. Assessment of lymphatic impairment and interstitial protein accumulation in patients with breast cancer treatment-related lymphedema using CEST MRI. Magnetic Resonance Medicine, 2016;75(1):345-55. https://doi: 10.1002/mrm.25649

15.) Mitsumori LM, McDonald ES, Neligan PC, Maki JH. Peripheral Magnetic Resonance Lymphangiography: Techniques and Applications. Techniques in Vascular and Interventional Radiology, 2016;19(4):262-272. https://doi: 10.1053/j.tvir.2016.10.007

16.) Bae JS, Yoo RE, Choi SH, Park SO, Chang H, Suh M, Cheon GJ. Evaluation of lymphedema in upper extremities by MR lymphangiography: Comparison with lymphoscintigraphy. Magnetic Resonance Imaging, 2018; 49:63-70. https://doi: 10.1016/j.mri.2017.12.024

17.) Cellina M, Oliva G, Menozzi A, Soresina M, Martinenghi C, Gibelli D. Non-contrast Magnetic Resonance Lymphangiography: an emerging technique for the study of lymphedema. Clinical Imaging, 2019; 53:126-13. https://doi: 10.1016/j.clinimag.2018.10.006

18.) Harvey N, Mayrovitz HN. Assessing Lower Extremity Lymphedema Using Upper and Lower Extremity Tissue Dielectric Constant Ratios: Method and Normal Reference Values. Lymphatatic Research Biology, 2019; 17(4):457-464. https://doi: 10.1089/1rb.2018.0039 19.) Tugral A, Viren T, Bakar Y. Tissue dielectric constant and circumference measurement in the follow-up of treatment-related changes in lower-limb lymphedema. International Angiology 2018; 37(1):26-31. https://doi: 10.23736/S0392-9590.17.03843-3

20.) Panici PB, Basile S, Maneschi F, Lissoni AA, Signorelli M, Scambia G, Angioli R, Tateo S, Mangili G, Katsaros D, Garozzo G, Campagnutta E, Donadello N, Greggi S, Melpignano M, Raspagliesi F, Ragni N, Cormio G, Grassi R, Franchi M, Giannarelli D, Fossati R, Torri V, Amoroso M, Crocè C, Mangioni C. Systematic pelvic lymphadenectomy vs. no lymphadenectomy 
in early-stage endometrial carcinoma: Randomized clinical trial. Journal of the National Cancer Institute, 2008; 100(23): 1707-1716. https://doi.org/10.1093/jnci/djn397

21.) The writing committee on behalf of the ASTEC Study Group (ASG). Efficacy of systematic pelvic lymphadenectomy in endometrial cancer (MRC ASTEC trial): A randomised study. The Lancet, 2009; 373(9658); 125-136. https://doi.org/10.1016/S0140-6736(08)61766-3

22.) Lécuru F, Mathevet P, Querleu D, Leblanc E, Morice P, Daraï E, Marret H, Magaud L, Gillaieau F, Chatellier G, Darget D. Bilateral negative sentinel nodes accurately predict absence of lymph node metastasis in early cervical cancer: Results of the SENTICOL study. Journal of Clinical Oncol. 2011; 29(13): 1686-1691. https://doi.org/10.1200/JCO.2010.32.0432

23.) Salvo G, Ramirez PT, Levenback CF, Munsell MF, Euscher ED, Soliman PT, Frumovitz M. Sensitivity and negative predictive value for sentinel lymph node biopsy in women with earlystage cervical cancer. Gynecol Oncol. 2017; 145(1): 96-101. https://doi.org/10.1016/j.ygyno.2017.02.005

24.) Tax C, Rovers MM., De Graaf C, Zusterzeel PL, Bekkers RL. The sentinel node procedure in early stage cervical cancer, taking the next step; a diagnostic review. Gynecol Oncol. 2015; 139(3): 559-567. https://doi.org/10.1016/j.ygyno.2015.09.076

25.) Mathevet P, Lécuru F, Magaud L, Bouttitie, F. Sentinel lymph node biopsy for early cervical cancer: Results of a randomized prospective, multicenter study (SENTICOL 2) comparing adding pelvic lymph node dissection vs. sentinel node biopsy only. Gynecol Oncol. 2017; 145(1): 2-3. https://doi.org/10.1016/j.ygyno.2017.03.029

26.) Niikura H, Okamoto S, Otsuki T, Yoshinaga K, Utsunomiya H, Nagase S, Takano T, Ito K, Watanabe M, Yaegashi N. Prospective study of sentinel lymph node biopsy without further pelvic 
lymphadenectomy in patients with sentinel lymph node-negative cervical cancer. Int. J Gynecol. Cancer, 2012; 22(7): 1244-1250. https://doi.org/10.1097/IGC.0b013e318263f06a

27.) Gortzak-Uzan L, Jimenez W, Nofech-Mozes S, Ismiil N, Khalifa MA, Dubé V, Rosen B, Murphy J, Laframboise S, Covens A. Sentinel lymph node biopsy vs. pelvic lymphadenectomy in early stage cervical cancer: Is it time to change the gold standard? Gynecol Oncol. 2010; 116(1):

\section{8-32. https://doi.org/10.1016/j.ygyno.2009.10.049}

28.) Lennox GK, Covens A. Can sentinel lymph node biopsy replace pelvic lymphadenectomy for early cervical cancer? Gynecol Oncol. 2017; 144(1): 16-20.

\section{https://doi.org/10.1016/j.ygyno.2016.08.337}

29.) Holloway RW, Ahmad S, Kendrick JE, Bigsby GE, Brudie L A, Ghurani GB, Stavitzski NM,

Gise JL, Ingersoll SB, Pepe JW. A prospective cohort study comparing colorimetric and fluorescent imaging for sentinel lymph node mapping inendometrial cancer. Annals of Surgical Oncol. 2017; 24(7):1972-1979. https://doi: 10.1245/s10434-017-5825-3

30.) Rossi EC, Kowalski LD, Scalici J, Cantrell L, Schuler K, Hanna RK, Method M, Ade M, Ivanova A, Boggess JF. A comparison of sentinel lymph node biopsy to lymphadenectomy for endometrial cancer staging (FIRES trial): A multicentre, prospective, cohort study. The Lancet Oncology, 2017; 18(3): 384-392. https://doi.org/10.1016/S1470-2045(17)30068-2

31.) Ridner SH, Doersam JK, Stolldorf DP, Dietrich MS. Development and Validation of the Lymphedema Symptom Intensity and Distress Survey-Lower Limb. Lymphatic Research and Biology, 2019; 16(6):538-546. https://doi: 10.1089/1rb.2017.0069

32.) Armer JM, Radina, ME, Porock D, Culbertson SD. Predicting breast cancer-related lymphedema using self-reported symptoms. Nursing Research, 2003; 52(6): 370-379. 
33.) Armer JM. The problem of post-breast cancer lymphedema: impact and measurement issues. Cancer Investigation, 2005; 23(1): 76-83. https://doi.org/10.1081/CNV-48707

34.) Armer JM, Stewart BR. A comparison of four diagnostic criteria for lymphedema in a postbreast cancer population. Lymphatic Research and Biology, 2005; 3(4): 208-217.

\section{https://doi.org/10.1089//rb.2005.3.208}

35.) Carter J, Raviv L, Appollo K, Baser R., Iasonos R, Barakat RR. A pilot study using the gynecologic cancer lymphedema questionnaire (GCLQ) as a clinical tool to identify lower extremity lymphedema in gynecologic cancer survivors. Gynecol Oncol. 2010; 117(2); 317-323. https://doi.org/10.1016/j.ygyno.2010.01.022

36.) Carter J, Huang HQ, Armer,J, Carlson JW, Lockwood S, Nolte S, Stewart BR, Kauderer J, Hutson A, Walker JL, Fleury AC, Bonebrake A, Soper JT, Mathews C, Zivanovic O, Richards WE, Tan A, Alberts, DS, Barakat RR, Wenzel,L. (2019). GOG 244 - The LymphEdema and Gynecologic cancer (LEG) study: The association between the gynecologic cancer lymphedema questionnaire (GCLQ) and lymphedema of the lower extremity (LLE). Gynecol Oncol. 2019; 155 (3): 452-460. https:// doi: 10.1016/j.ygyno.2019.09.027

37.) Yost KJ, Cheville, AL, Weaver AL, Al Hilli, M, Dowdy SC. Development and validation of a self-report lower-extremity lymphedema screening questionnaire in women. Physical Therapy, 2013; 93(5): 694-703. https://doi.org/10.2522/ptj.20120088

38.) Paskett ED, Caan BJ, Johnson L, Bernardo BM, Young GS, Pennell ML, Ray RM, Kroenke CH, Porter PL, Anderson GL. The Women's Health Initiative (WHI) life and longevity after cancer (LILAC) study: Description and baseline characteristics of participants. Cancer Epidemiology Biomarkers \& Prevention. 2018; 27(2): 125-37. https://doi.org/10.1158/1055-9965.EPI-17-0581 